\title{
BMJ Open Using a behavioural approach to explore the factors that affect questionnaire return within a clinical trial: a qualitative study based on the theoretical domains framework
}

\author{
Louisa Lawrie (D) , Eilidh M Duncan, Jennifer Dunsmore, Rumana Newlands, \\ Katie Gillies
}

To cite: Lawrie L, Duncan EM, Dunsmore J, et al. Using a behavioural approach to explore the factors that affect questionnaire return within a clinical trial: a qualitative study based on the theoretical domains framework. BMJ Open 2021;11:e048128. doi:10.1136/ bmjopen-2020-048128

- Prepublication history and additional supplemental materials for this paper is available online. To view these files, please visit the journal online (http://dx.doi.org/10. 1136/bmjopen-2020-048128).

Received 28 December 2020 Revised 08 March 2021 Accepted 24 March 2021
Check for updates

(C) Author(s) (or their employer(s)) 2021. Re-use permitted under CC BY-NC. No commercial re-use. See rights and permissions. Published by BMJ.

Health Services Research Unit, University of Aberdeen College of Life Sciences and Medicine, Aberdeen, UK

Correspondence to

Dr Louisa Lawrie;

louisa.lawrie1@abdn.ac.uk

\section{ABSTRACT}

Objectives To identify barriers and enablers to participant retention in trials requiring questionnaire return using the theoretical domains framework (TDF).

Study design and setting We identified and subsequently invited participants who did not return at least one questionnaire during their participation in a clinical trial for one-to-one semi-structured telephone interviews. We used a behavioural framework (TDF) to explore whether any of the behavioural domains (eg, beliefs about consequences, emotion) affected questionnaire return. Thereafter, we generated a series of belief statements which summarised the content of participants' main responses and coded these under separate themes.

Participants We distributed invites to 279 eligible individuals and subsequently interviewed 9 participants who took part in the $\mathrm{C}$-Gall trial. The $\mathrm{C}$-Gall trial required participants to complete five postal questionnaires during their participation.

Results Nine participants were interviewed. We developed 7 overarching themes which were relevant for returning postal questionnaires and identified both barriers and enablers from 11 core domains: knowledge; beliefs about consequences; environmental context and resources; reinforcement; emotion; beliefs about capabilities; behavioural regulation; social professional role and identity; skills; intentions and goals. Relevant content coded under these salient domains were categorised into seven key themes: unclear expectations of trial participation, personal attributes for questionnaire return, commitment to returning questionnaires given other priorities, sources of support in returning the questionnaires, individual preferences for presentation mode and timing of the questionnaires, internal and external strategies to encourage questionnaire return and the significance of questionnaire non-return.

Conclusion We demonstrate how a behavioural approach may be useful for clinical trials associated with significant participation burden (e.g. trials that require multiple questionnaire responses), acting as the essential groundwork for the development of appropriate evidencebased solutions to combat retention issues.

Trial registration number 55215960; Pre-results.

\section{Strengths and limitations of this study}

- We used an established theoretical framework to explore the factors that influence questionnaire nonresponse among clinical trial participants.

- It was difficult to engage trial non-responders and thus we recruited a small purposive sample $(n=9)$.

- Findings, and the overall approach, will be useful for trialists to consider and adapt according to their clinical context.

\section{INTRODUCTION}

Postal and electronic questionnaires are commonly used to obtain outcome data from participants within randomised controlled trials. While this method of data collection can be both practical and relatively inexpensive, researchers often place heavy reliance on participants to successfully complete and return questionnaires. Trial retention rates drop when participants fail to return questionnaires, and this form of loss to follow-up poses a significant threat to the integrity of clinical trial findings. For example, in the presence of incomplete or lost questionnaire data, researchers are faced with the harsh reality that their findings may be tainted by a reduction in statistical power and the possibility that their results are biased if participants who provide data differ in some way from non-responders. ${ }^{1}$ The challenges associated with high attrition rates collectively undermine the extent to which clinical trial findings can guide the implementation of the best evidence-based healthcare interventions. The marked disadvantages associated with high attrition rates has led clinical unit trial directors across the UK to identify 'methods to minimise attrition' as a top priority. ${ }^{2}$ 
A recent survey conducted with key trial stakeholders to investigate the prioritisation of unanswered research questions around retention (the PRioRiTy II study) identified the number one ranked question as "What motivates a participant's decision to complete a clinical trial? ${ }^{3}$ Trial retention often involves several behaviours such as attending a clinic appointment or returning a questionnaire. Using a behavioural approach to investigate the factors that influence retention appears to be a promising avenue for facilitating a science of retention. This approach would involve analysing the applicability and explanatory power of key concepts from behavioural science to the target behaviour which influences trial retention. Prior studies have adopted a behavioural lens to investigate the origins of other behaviours within clinical trials, such as the implementation of a treatment arm and recruitment of participants. ${ }^{45}$ Yet, there is a marked absence of studies that adopt a behavioural science approach to investigate the factors that influence retention. ${ }^{67}$

In the present qualitative interview study, we aimed to uncover the factors that influenced questionnaire response rates using the theoretical domains framework (TDF) to investigate the barriers and enablers to questionnaire return within a clinical trial. The TDF is an established framework that integrates 33 theories of behaviour into 14 domains that inhibit or enable behaviour (knowledge, skills, social/professional role and identity, beliefs about capabilities, beliefs about consequences, optimism, reinforcement, intentions, goals, memory/attention/decision processes, environmental context and resources, social influences, emotion and behavioural regulation) ${ }^{8}$ We applied the TDF within the design of this study to assess questionnaire return. The TDF was originally designed to understand and improve implementation of an intervention, ${ }^{9}$ and it has largely been applied to health professional behaviours. ${ }^{10-12}$ However, a growing body of literature has demonstrated the TDF's application to the behaviour of the general public and patients. ${ }^{1314}$ The current study extends this literature and demonstrates how the TDF can be applied to investigate patient behaviour within clinical trials.

\section{METHODS}

\section{Design}

This was a qualitative study, using semi-structured oneto-one interviews, which was embedded within the C-Gall clinical trial (ISRCTN: 55215960, see box 1). The topic

\section{Box 1 Description of the C-Gall trial}

The C-Gall trial is a randomised controlled trial comparing laparoscopic cholecystectomy with conservative management for preventing recurrent symptoms and complications in adults with uncomplicated symptomatic gallstones. Participation in the trial includes completing five postal questionnaires at: 3, 6, 9, 12 and 24 months. guide (see online supplemental appendix 1 ) was informed by the TDF and refined by the research team and Public and Patient Involvement (PPI) members. In addition to interviews conducted as part of this embedded project, interviews conducted with C-Gall participants as part of a broader project (the Systematic Techniques to Enhance Retention in RCTs, STEER, study) exploring trial retention across several trials including C-Gall were also included in this study during analysis. ${ }^{15}$ The STEER study developed the topic guide for use in the interviews. The topic guide was iteratively updated to ensure robustness.

\section{Patient and public involvement}

As part of the STEER study, two public partners reviewed the interview topic guide to ensure the questions were clear and acceptable for the purpose of the study. Patients or the public were not actively involved in the reporting or dissemination plans of the research.

\section{Participants}

We invited a purposive sample of participants who had not returned at least one questionnaire at any time point during the study, and therefore had discontinued their follow-up at least once. An invitation letter and participant information leaflet were distributed to all potential participants with a reply slip and a prepaid envelope. A researcher $(\mathrm{JD} / \mathrm{RN})$ then contacted interested participants to discuss the study further and book a mutually convenient time for a telephone interview. All invitation packs were distributed from the C-Gall trial office to maintain the confidentiality of potential participants. Two attempts were made to engage with potential participants. One hundred and sixty-five C-Gall participants were invited to participate in this phase of the interview study and an earlier 114 had been invited as part of the STEER study.

\section{Data collection}

Interviews were conducted by RN (academic researcher, female, age 40 years) and JD (research assistant, female, age 25 years) to explore participants' experiences of the trial, reasons for not returning questionnaires and the behavioural barriers and facilitators to questionnaire return. Data collection was conducted between January and November 2019. Participants were made aware that the interviewers were neither clinicians nor involved in the day-to-day conduct of the C-Gall trial. The researchers obtained verbal informed consent from participants. All interviews were audio-recorded and transcribed verbatim by an external transcribing service. We judged the sufficiency of our sample size against five key aspects of information power: whether the study aim is broad or narrow (with a focused aim requiring a smaller sample); dense or sparse sample specificity (where dense specificity requires a smaller sample); application or not of established theory (theoretical perspectives requiring smaller sample); quality of the dialogue (with strong clear communication 
requiring less); and finally, whether case or cross-case analysis (with cross-case requiring more participants). ${ }^{16}$

\section{Data analysis}

A TDF coding guide was used to aid interpretation: this was developed and iteratively updated during the coding process (RN, LL, EMD, KG). Three of the nine interview transcripts were triple coded independently (LL, KG, EMD) prior to comparing the coding results. The few coding discrepancies identified during this process were discussed to reach consensus. Specialist input from a TDF expert (EMD) was used to resolve coding disagreements in instances where it was difficult to reach a consensus. One researcher (LL) coded all participants' interview responses into the relevant theoretical domains.

After coding data into theoretical domains, belief statements were generated (LL) which represented similar underlying beliefs held by participants within each domain. ${ }^{17}$ Belief statements were designed to elicit detail on the role of each domain in influencing the behaviour (questionnaire return). Statements were worded to capture multiple utterances that conveyed a similar meaning. Specific beliefs that centralised on the same theme or represented the opposite were grouped together under the same statement.

We used the recommended procedure for TDF analysis to identify the relevant domains that were most likely to influence the behaviour ${ }^{17}$ : (1) the frequency of belief statements across all domains; (2) the presence and prevalence of conflicting beliefs; and (3) evidence of strong beliefs that influence the behaviour. All three criteria were considered concurrently to judge the relevance of each domain. We developed overarching themes that described the content of related belief statements and domains to effectively summarise the findings. Overarching themes were initially generated by LL who assessed the belief statements across all domains and categorised them into separate themes. These themes and belief statements were refined by KG and EMD to ensure that they summarised the data accurately.

\section{RESULTS}

\section{Sample characteristics}

Nine participants in total were recruited. All interview participants were participating in the C-Gall trial at the time of interview, six were recruited specifically for the purposes of the C-Gall trial substudy and three recruited via the STEER study. Participant demographics are

\begin{tabular}{lc}
\hline \multicolumn{2}{l}{ Table 1 Sample characteristics } \\
\hline Characteristic & (n/9) \\
\hline Gender & \\
$\quad$ Male & $2 / 9$ \\
Female & $7 / 9$ \\
\hline Median age (years, range) & 53 years (35-75 years) \\
\hline
\end{tabular}

provided in table 1 . This is comparable to the entire population of C-Gall trial non-responders (in February 2020) whom were $79.4 \%$ women and $20.6 \%$ men with a median age of 51 . The length of interviews ranged from $25 \mathrm{~min}$ to 1 hour $28 \mathrm{~min}$ (median=36 min).

\section{Overall findings}

The following sections describe the domains that were relevant to the target behaviour (questionnaire return). Domains and associated belief statements were categorised into overarching themes to summarise the main messages arising from the data. A total of seven themes were identified. These were: unclear expectations of trial participation; personal attributes for questionnaire completion; commitment to returning questionnaires given other priorities; the significance of questionnaire non-return; individual preferences for presentation mode and timing of the questionnaires; internal and external strategies to encourage questionnaire return and sources of support in returning the questionnaires. Each of these themes will be presented in detail below. An extended table containing the frequency of the TDF domains and belief statements is provided in online supplemental appendix 2. Figure 1 represents a visual illustration of the findings. A summary table of the findings is also presented in online supplemental appendix 3.

\section{Theme 1: unclear expectations of trial participation}

The majority of participants reported being unaware of the duration of their participation within the trial, although some indicated a certain level of awareness of their ongoing participation.

I think at the appointment initially, they said that the trial had been extended. They didn't give me a timeframe for how long it would go on for. (Participant 1)

Importantly, participants suggested that the activities involved in the trial did not meet their initial expectations, with some indicating that they expected a greater level of medical attention, such as support and information provided to help manage their condition, such as dietary advice, among participants randomised to conservative management.

I'm not sure if it's just myself or if other people are on the same, medical management (conservative management), a little bit more information, a little bit more contact with someone asking, 'How are you doing? Have you received this information?' or just giving information out would help a bit more. I'm not sure if it's... if you don't do it, I didn't know that medical management was just filling in questionnaires. I thought there'd be a little bit more to it, that's all. (Participant 8)

Most participants indicated that they did not perceive the questions within the questionnaire as relevant to their circumstances. This was because they were either not experiencing pain at the time of receiving the questionnaire or 


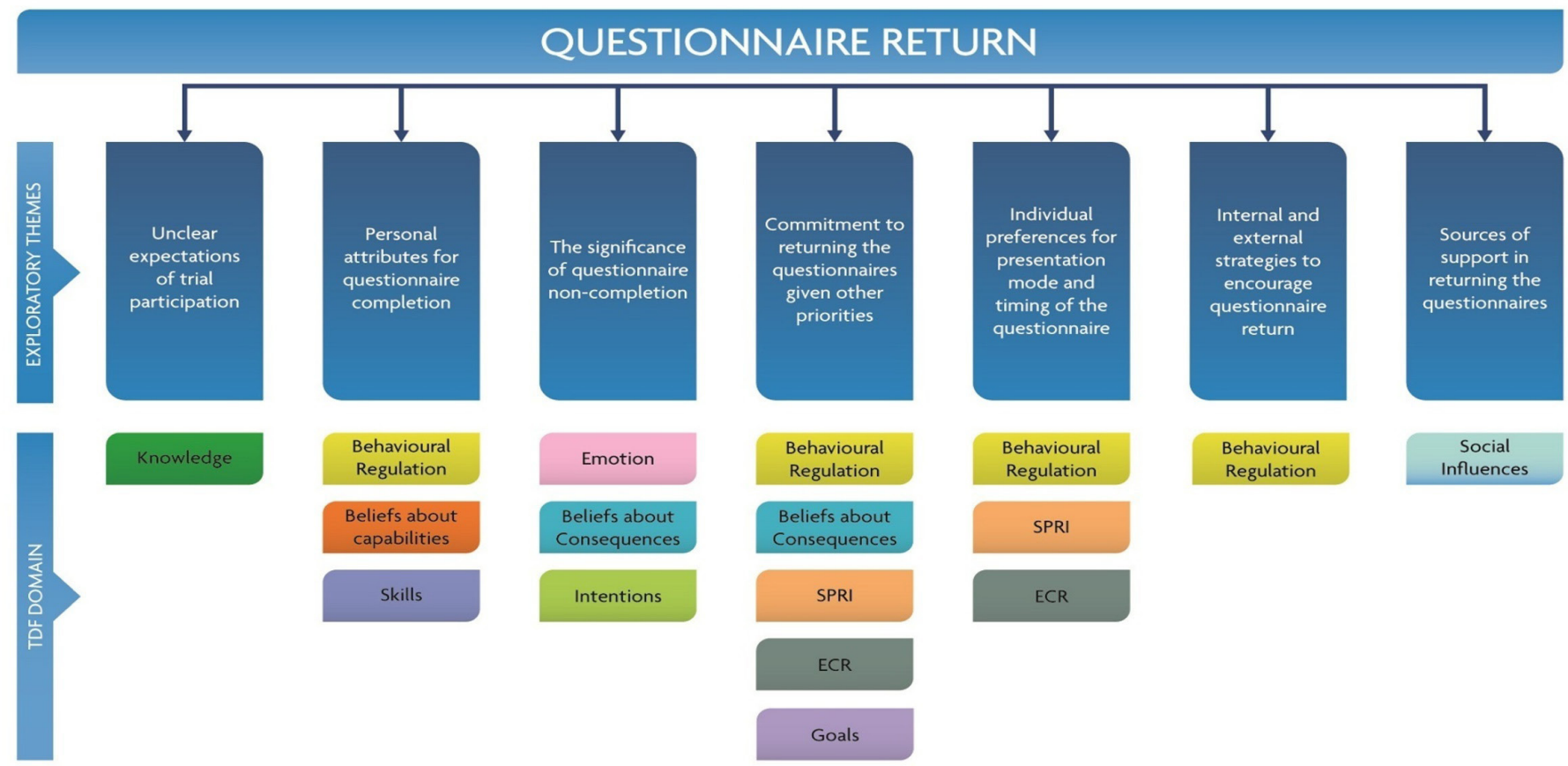

Figure 1 Overarching themes and associated TDF domains. ECR, environmental context and resources; SPRI, social professional role and identity; TDF, theoretical domains framework.

because they had been assigned to conservative management and therefore did not receive gallstone surgery and regarded the questions as irrelevant.

Well, there were a few. I mean, I started off well and then because nothing had happened... I hadn't had any pain or anything and then I'd put them aside and then somebody would send another one and say, 'You haven't completed it'. (Participant 2)

Participants also reported confusion over receiving the same questionnaire on multiple occasions. As they did not expect to receive (or understand the purpose of) identical questionnaires at different time points, they did not send some of the questionnaires back to the trial office.

\section{Theme 2: personal attributes for questionnaire completion}

The majority of participants recognised that there were certain skills required to complete the questionnaires, such a remembering the level of pain experienced over a certain amount of time.

No, I suppose you have to be able to read, you have to be able to understand, take the time and comprehend what it's asking you as well. And also, to recollect, to recall when you've gone to the doctors, all that sort of thing, you know? (Participant 7)

Some also acknowledged that completing the questionnaire required concentration, and sometimes this was reported as a barrier to completion and return.

I suppose if the questions had been really complicated or difficult to answer, maybe. Or if you just can't remember what exactly has happened over the time. I suppose the time between questionnaires, if it's every three months then that's not too bad, but if it was longer than that then I'd definitely have trouble remembering exactly what symptoms I'd had and how many times and all the fine details. (Participant 1)

\section{Theme 3: the significance of questionnaire non-return}

Participants reported feelings of guilt triggered by questionnaire non-return. This was mostly linked to the belief that they were letting the trial team down and having a negative effect on trial results.

It's been playing on my mind that I haven't done it yet. Not willing me but making me angry with myself for not doing it...For messing up the trials. (Participant 3)

Around half of the participants reported a sense of satisfaction brought about by successfully completing and returning the questionnaires and felt it was their duty to complete this task as a trial participant. Feelings of satisfaction were mostly linked to their beliefs about consequences: that their contribution would help the research.

Well, you've completed a job, you know that it's important, it helps in clinical trials, it's helping finding out stuff. That's the upside of doing it, and you feel satisfaction, that you're making a difference. (Participant 4)

\section{Theme 4: commitment to returning the questionnaires given} other priorities

Some participants suggested that they were not committed to performing the behaviour because they did not prioritise completion and return among other duties and 
responsibilities. Sometimes participants reported being too busy to schedule in completion and return.

The questionnaire thing for me is a barrier because I'll think, 'Oh, I'll put it to the side, I'll do it when I've got time,' and I never get the time. I've got my second one to fill and it's been there for months. Really bad! (Participant 2)

On the contrary, a few participants stated their commitment to completing the questionnaires because they had agreed to take part in the trial and they recognised the negative consequences of not returning the questionnaires, such as wasting the researcher's time.

Well, I want to return it so it is quite important, because then I'm wasting your time and money by you sending these forms out if I'm not going to bother. (Participant 9)

\section{Theme 5: individual preferences for presentation mode and timing of the questionnaires}

Preference for questionnaire format differed among participants: some regarded postal administration as convenient for them, others indicated that they would have preferred to speak to someone or complete the questionnaire electronically.

I much preferred it when I could give oral answers to a simplified version in which she said, "These are just the main questions that we need to know." That didn't take nearly as long, and it was quite focused and straightforward. (Participant 2)

Some participants linked their preference for completing the questionnaire with a trial member of staff to their social personality. The majority of participants perceived the questionnaire to be boring, and most indicated that if it were a lengthy questionnaire, they would struggle to complete it.

It wasn't very interesting, it was pretty boring. (Participant 3)

\section{Theme 6: internal and external strategies to encourage questionnaire return}

Some participants indicated strategies that they had used to help them complete the questionnaires. These included 'internal' strategies such as making a plan to complete and return the questionnaires and keeping a note of symptoms to ensure accurate reporting.

I would actually think, "Well, I've got to get this done" and do it and then send it off. Saying that, yes, probably... You're right, I would probably say, "Oh, I'm going to sit down this evening, fill this questionnaire in and then get it sent off'." So, yes, in that way, there was a plan. (Participant 5)

Some participants indicated that they completed the questionnaires whenever they had spare time and would often complete the questionnaire as soon as they received it to avoid forgetting about it.

Yes, like I say, once I received it, I filled it in and on the way to pick my son up from school I put it in a post box. (Participant 8)

Participants also mentioned external strategies to encourage questionnaire return. The majority of participants reported that they did not expect to receive any incentives or rewards for completing and returning the questionnaires, and for one participant rewards/incentives offered would have been perceived as a form of bribery:

Yeah, I just want to do it because I want to do it. I don't really want anybody to try and bribe me to do it or encourage me to do it due to any kind of incentive, no. In some ways, actually, that would put me off. (Participant 3)

Most participants suggested that they would have liked to receive a prompt or reminder to complete and return the questionnaires.

It's an extra thing that we volunteered to do, I could have just said no to my surgery anyway and not gone for this, do you understand what I mean, because I had that choice. But I went for this and I volunteered for it, just that little bit of a reminder and a bit of support, "We're helping you out on this, it's okay, I know it's late but can you try and get it in now. Or do you want to come and see me?" There's a few more options. That's it. (Participant 6).

However, one participant indicated that they had received a friendly reminder from the trial office and believed it to be effective because it was not communicated in a forceful manner.

I think once I didn't return it and they sent me out a reminder questionnaire, which was good. It wasn't a telling off, but it was just a reminder that it was there. That was good if you know what I mean...Yeah, without being forceful, kind of thing. (Participant 4)

\section{Theme 7: sources of support in returning the questionnaires}

A couple of participants reported that they received support from family members to return the questionnaires, while the majority indicated that they completed and returned the forms independently. All participants indicated that they received some level of support from the trial office to complete trial-related activities. This included receiving gentle reminders or facilitating questionnaire completion over the phone with a trial staff member.

Once I've filled it in, my husband supports me in terms of he gets it to you, as in he posts it for me because I don't go out to post it when I've got work or whatever. But that's the only support...my husband 
is understanding. He understands the pain and he helps me when I'm suffering and stuff, but no, I don't have any other support, not really. (Participant 6)

Participants suggested that they did not feel any pressure to complete and return the questionnaires, and for some this was a barrier to questionnaire completion and return.

No, I've got no pressures from anyone. Nobody encourages me either. At first, my sister half encouraged me to take part because I was about $90 \%$ sure I was going to do it, and then after talking to her and a couple of others, just things that were said I just said, "Yeah, I'll do it". (Participant 1)

\section{DISCUSSION}

This study investigated trial retention using a behavioural science approach: we applied a theoretical framework to understand the barriers and enablers to questionnaire return within a clinical trial. Obtaining maximum questionnaire response rates is essential to maintain scientific validity, reduce bias and optimise trial rigour. Our findings suggest that many behavioural domains from the TDF were relevant and important for influencing questionnaire return and as such this behavioural approach lent itself well to the problem of trial retention. We believe the TDF was particularly suited to investigating the factors that influence questionnaire return, given that this is a very specific behaviour characterised by low complexity. This allowed us to isolate the factors that influence this specific behaviour. These findings may be transferable to other trials with postal questionnaires used as a mechanism for data collection, however, it may be difficult to apply the findings of the current study to other trial retention behaviours, such as attending a clinic appointment. Identifying the relevant influential domains within the TDF is an essential stage required prior to developing behaviour change interventions designed to improve retention.

One of the most frequently reported barriers to questionnaire return was related to the fact that the trial did not meet the participants' expectations, either because they expected to receive additional medical attention or they did not understand how the questionnaire related to their circumstances (e.g. due to a lack of pain/symptoms experienced or because they had not undergone surgery). Some studies suggest that it is not uncommon for trial staff to communicate inaccuracies about the timing and delivery of study questionnaires within initial clinical trial consultations, and the right to withdraw is sometimes highlighted without explaining the associated negative consequences. ${ }^{18}$ Likewise, a recent study demonstrated that withdrawal and retention is often poorly described within participant information leaflets. ${ }^{19}$ Our study suggests that retention may drop when expectations are violated, particularly when a mismatch exists between the initial briefing of the trial and participants' actual experience. This highlights the importance of managing expectations, clarifying the activities involved within the trial and how this relates to study outcomes. Relatedly, many participants acknowledged that questionnaire completion required concentration, engaged memory processes and demanded literacy and communication skills. It may be helpful for trial staff to outline the requirements of trial activities in terms of the expected duration and methods participants can use to facilitate questionnaire return.

Participants reported a mixed level of commitment devoted to completing and returning the questionnaire, with some indicating high levels of commitment due to the recognised consequences of failing to return the questionnaires, while others admitted that their lack of commitment to completing the questionnaires influenced memory and prioritisation. In a study assessing techniques to increase retention in a behavioural weight loss trial, one technique deployed involved communicating the consequences of trial drop-out to participants, which included how attrition might bias findings. ${ }^{20}$ This appeared to be an effective method of increasing retention and could be a technique applied to increase motivation and commitment to clinical trials.

In terms of social support, a few participants indicated that their family members helped them to return the questionnaires whereas others suggested that they completed and returned the questionnaires independently. Although not necessarily perceived to be a barrier to retention within this study, some studies indicate that a lack of social support influences retention-related behaviours, such as erratic clinic attendance. ${ }^{21}$ It may be useful for trial staff to be mindful of the importance of social support for each participant, and where unavailable, offer additional support to those who require it, particularly those who may have physical/mental disabilities that hinder trial participation. Providing support to participants where required may also help to ensure that trials are more inclusive.

In response to questions related to questionnaire format, participants reported mixed preferences: some indicated that postal administration was ideal, whereas others suggested that they preferred to complete the questionnaire electronically or over the phone. It may be helpful for trial staff to ask participants their preferred mode of questionnaire delivery, and while it may not be practical to tailor the administration format for each participant, it could reduce the number of reminders required for distribution and enhance retention. It is also important to note that there may be generational differences in preferences for questionnaire format, where older adults may be more likely to prefer paperbased or telephone forms as opposed to electronic versions. ${ }^{22}$ Likewise, some individuals may not have access to electronic devices and considerations of digital poverty should be taken into account. Tailoring the mode of administration to participants' preferences and 
circumstances may help to include the groups of people that are commonly under-represented in UK medical research. ${ }^{23}$

Participants reported mixed strategies used to support questionnaire return, which included internal strategies that represented personal behavioural regulatory practices used to facilitate behaviour, such as making a plan to complete and return the questionnaires and maintaining a diary of symptoms to simplify questionnaire completion. External strategies reported to be helpful included reminders distributed from the trial office. This finding supports the evidence which suggests that the use of electronic prompts can improve questionnaire response rates. ${ }^{24}$ Contrary to the evidence which suggests the effectiveness of monetary incentives ${ }^{25}$ participants indicated that incentives or rewards would have actually been perceived as a form of bribery and would deter participation from the trial. Participants are likely to have different preferences for the type of reward/incentive received, and these need to be designed carefully to ensure that they are not coercive and do not encourage trial participation for the wrong reasons. ${ }^{26}$

We have demonstrated how a behavioural approach can be applied to assess the factors that influence a key trial retention behaviour: return of a postal questionnaire. We performed a behavioural diagnosis which is the first stage of designing TDF informed interventions that can improve retention. Effective interventions often contain behaviour change techniques (BCTs) that comprise the 'active ingredients' designed to elicit change. By assessing the relevance of each behavioural domain within the TDF, effective BCTs can be matched and incorporated within the intervention to facilitate behaviour change. ${ }^{9}$ One study has demonstrated how a theory informed cover letter containing BCTs can improve retention. ${ }^{7}$ Another study suggests that is not uncommon for studies to apply BCTs implicitly in an effort to elicit change and improve trial participation. ${ }^{6}$ Our study suggests that an in-depth qualitative analysis can be useful for identifying participant-relevant BCTs that can be considered to improve retention. We have designed a TDF informed cover letter and newsletter with embedded BCTs that are likely to be effective based on the interview findings reported within this paper. These will be distributed to C-Gall trial participants in an effort to boost the questionnaire return rate. We have also outlined practical considerations other researchers may wish to take into account to increase questionnaire return rate, such as managing participants' expectations of trial-related activities (e.g. how many questionnaires they will be expected to complete), highlighting the negative consequences of participant drop-out, tailoring the administration of questionnaires to suit individual preferences and circumstances and providing support where required. These adjustments are similar to the recommendations outlined in a recent paper. ${ }^{27}$

\section{Strengths and weaknesses}

We used a validated theoretical framework to guide the design and analysis phases of this qualitative study. It was appropriate to use the TDF in this instance because we were investigating the barriers and enablers to conducting a specific retention-related behaviour: questionnaire return. Although we distributed invites to over 300 potential participants, we only secured 9 interview participants. Our sample size was relatively small as it was difficult to engage trial non-retainers. It is possible that additional themes may have emerged if we had interviewed more participants. However, given our findings resonate with other literature on participant reported reasons for challenges in trial retention, ${ }^{28}$ we believe many of the key factors were identified. We were also satisfied that our sample size was sufficient as no new concepts were identified within the TDF domains over the last three interviews. This is in line with stopping criterion for theory informed interviews and the guidance on information power. ${ }^{16}$ Nevertheless, our findings may be limited to a particular trial context, specifically those that require participants to respond to several questionnaires. Other trials may require participants to attend a follow-up clinic appointment or to adhere to certain medication. The applicability of our findings may not necessarily be extended to other non-retention behaviours.

\section{Conclusion}

Our study uncovers the common barriers and enablers to return of postal questionnaires in clinical trials using a behavioural framework. We demonstrate how a behavioural approach may be particularly useful for clinical trials associated with significant participation burden (e.g. trials that require multiple questionnaire responses), acting as the essential groundwork for the development of appropriate evidence-based solutions to combat retention issues. Findings will serve as a guiding framework when designing trials to limit barriers and enhance enablers of retention, complementing host trial data and contributing to improving trial methodology.

\section{Twitter Louisa Lawrie @LawrieLouisa and Katie Gillies @GilliesKatie}

Acknowledgements The authors would like to thank the patients for volunteering their time to participate in this study and to Rebecca Bruce and Karen Innes for facilitating recruitment.

Contributors LL: methodology, formal analysis, writing —original draft, review and editing, visualisation and project administration. EMD: methodology, formal analysis, writing —review and editing, visualisation and supervision. JD: methodology, investigation, writing - review and editing and project administration. RN: methodology, investigation, writing — review and editing and project administration. KG: conceptualisation, methodology, formal analysis, writing—review and editing and supervision.

Funding This research is funded by the Chief Scientist Office of the Scottish Government's Health and Social Care Department (HIPS/16/46). KG held a Medical Research Council UK Methodology Fellowship during the delivery of this project (MR/L01193X/1). The Health Services Research Unit, Institute of Applied Health Sciences (University of Aberdeen), is core-funded by the Chief Scientist Office of the Scottish Government Health and Social Care Directorates. The funders had no involvement in study design, collection, analysis and interpretation of data, reporting or the decision to publish. 
Competing interests None declared.

Patient consent for publication Not required.

Ethics approval This study was approved through the parent trial from NHS North of Scotland Research Ethics Committee (16/NS/0053). Informed consent was obtained from all participants.

Provenance and peer review Not commissioned; externally peer reviewed.

Data availability statement All data relevant to the study are included in the article or uploaded as supplementary information. All data relevant to the study are included in the article.

Supplemental material This content has been supplied by the author(s). It has not been vetted by BMJ Publishing Group Limited (BMJ) and may not have been peer-reviewed. Any opinions or recommendations discussed are solely those of the author(s) and are not endorsed by BMJ. BMJ disclaims all liability and responsibility arising from any reliance placed on the content. Where the content includes any translated material, BMJ does not warrant the accuracy and reliability of the translations (including but not limited to local regulations, clinical guidelines, terminology, drug names and drug dosages), and is not responsible for any error and/or omissions arising from translation and adaptation or otherwise.

Open access This is an open access article distributed in accordance with the Creative Commons Attribution Non Commercial (CC BY-NC 4.0) license, which permits others to distribute, remix, adapt, build upon this work non-commercially, and license their derivative works on different terms, provided the original work is properly cited, appropriate credit is given, any changes made indicated, and the use is non-commercial. See: http://creativecommons.org/licenses/by-nc/4.0/.

\section{ORCID iDs}

Louisa Lawrie http://orcid.org/0000-0002-9867-2184

Katie Gillies http://orcid.org/0000-0001-7890-2854

\section{REFERENCES}

1 De ED. Reducing missing data in surveys: an overview of methods. Vol. 35, 2001.

2 Smith CT, Hickey H, Clarke M, et al. The trials methodological research agenda: results from a priority setting exercise, 2014. Available: http://www.trialsjournal.com/content/15/1/32

3 Brunsdon D, Biesty L, Brocklehurst P, et al. What are the most important unanswered research questions in trial retention? A James lind alliance priority setting partnership: the priority II (prioritising retention in randomised trials) study. Trials 2019;20:593.

4 Presseau J, Mutsaers B, Al-Jaishi AA, et al. Barriers and facilitators to healthcare professional behaviour change in clinical trials using the theoretical domains framework: a case study of a trial of individualized temperature-reduced haemodialysis. Trials 2017;18:227.

5 Lalu MM, Foster M, Presseau J, et al. What are potential barriers and enablers to patient and physician participation in Canadian cell therapy trials for stroke? A stakeholder interview study. BMJ Open 2020;10:e034354.

6 Duncan EM, Bennett T, Gillies K. Assessing effective interventions to improve trial retention: do they contain behaviour change techniques? Trials 2020;21:213.

7 Goulao B, Duncan A, Floate R, et al. Three behavior change theoryinformed randomized studies within a trial to improve response rates to trial postal questionnaires. J Clin Epidemiol 2020;122:35-41.

8 Cane J, O'connor D, Michie S. Validation of the theoretical domains framework for use in behaviour change and implementation research, 2012. Available: http://www.implementationscience.com/content/7/ $1 / 37$
9 Atkins L, Francis J, Islam R, et al. A guide to using the theoretical domains framework of behaviour change to investigate implementation problems. Implement Sci 2017;12:77.

10 Sargent L, McCullough A, Del Mar C, et al. Using theory to explore facilitators and barriers to delayed prescribing in Australia: a qualitative study using the theoretical domains framework and the behaviour change wheel. BMC Fam Pract 2017;18:20.

11 Duncan EM, Francis JJ, Johnston M, et al. Learning curves, taking instructions, and patient safety: using a theoretical domains framework in an interview study to investigate prescribing errors among trainee doctors. Implement Sci 2012;7.

12 Islam R, Tinmouth AT, Francis JJ, et al. A cross-country comparison of intensive care physicians' beliefs about their transfusion behaviour: A qualitative study using the theoretical domains framework. Implement Sci 2012;7.

13 Shaw RL, Holland C, Pattison HM, et al. Patients' perceptions and experiences of cardiovascular disease and diabetes prevention programmes: a systematic review and framework synthesis using the theoretical domains framework. Soc Sci Med 2016;156:192-203.

14 Hallsworth K, Dombrowski SU, McPherson S, et al. Using the theoretical domains framework to identify barriers and enabling factors to implementation of guidance for the diagnosis and management of nonalcoholic fatty liver disease: a qualitative study. Transl Behav Med 2020;10:1016-30.

15 Gillies K, Bower P, Elliott J, et al. Systematic techniques to enhance rEtention in randomised controlled trials: the steer study protocol. Trials 2018;19:197.

16 Malterud K, Siersma VD, Guassora AD. Sample size in qualitative interview studies: guided by information power. Qual Health Res 2016;26:1753-60.

17 Atkins L, Francis J, Islam R, et al. A guide to using the theoretical domains framework of behaviour change to investigate implementation problems. Implement Sci 2017;12:77.

18 Tunji-Ajayi P, Duncan EM, Gillies K. An embedded mixed-methods study highlighted a lack of discussions on retention in clinical trial consultations. J Clin Epidemiol 2020;123:49-58.

19 Kearney A, Rosala-Hallas A, Bacon N, et al. Reducing attrition within clinical trials: the communication of retention and withdrawal within patient information leaflets. PLoS One 2018;13:e0204886.

20 Goldberg JH, Kiernan M. Innovative techniques to address retention in a behavioral weight-loss trial. Health Educ Res 2005;20:439-47.

21 Bender BG, Ellison MC, Gleason M, et al. Minimizing attrition in a long-term clinical trial of pediatric asthma. Ann Allergy Asthma Immunol 2003;91:168-76.

22 Bech M, Kristensen MB. Differential response rates in postal and web-based surveys among older respondents, 2009. Available: http://www.surveymethods.org

23 Smart A, Harrison E. The under-representation of minority ethnic groups in UK medical research. Ethn Health 2017;22:65-82.

24 Clark HD, Graham ID, Karovitch A, et al. Do postal reminders increase postpartum screening of diabetes mellitus in women with gestational diabetes mellitus? A randomized controlled trial. Am J Obstet Gynecol 2009;200:634.e1-e7.

25 Yu S, Alper HE, Nguyen A-M, et al. The effectiveness of a monetary incentive offer on survey response rates and response completeness in a longitudinal study. BMC Med Res Methodol 2017;17:77.

26 Vellinga A, Devine C, Ho MY, et al. What do patients value as incentives for participation in clinical trials? A pilot discrete choice experiment. Res Ethics 2020;16:1-12.

27 Newlands R, Duncan E, Presseau J. Why trials lose participants: a multi-trial investigation of participants' perspectives using the theoretical domains framework. J Clin Epidemiol 2021 doi:10.1016/j. jclinepi.2021.03.007

28 Skea ZC, Newlands R, Gillies K. Exploring non-retention in clinical trials: a meta-ethnographic synthesis of studies reporting participant reasons for drop out. BMJ Open 2019;9:e021959. 